\title{
Autologous cryopreserved leukapheresis cellular material for chimeric antigen receptor $-T$ cell manufacture
}

\author{
SESHU TYAGARAJAN ${ }^{1}$, DAVID SCHMITT ${ }^{2}$, CHRISTOPHER ACKER $^{1} \&$ ERIK RUTJENS $^{2}$ \\ ${ }^{1}$ Novartis Pharmaceuticals Corporation, East Hanover, New fersey, USA, and ${ }^{2}$ Novartis Pharma AG, Basel, \\ Switzerland
}

\begin{abstract}
Tisagenlecleucel, a CD19-specific autologous chimeric antigen receptor (CAR)-T cell therapy, is efficacious for the treatment of relapsed/refractory B-cell precursor acute lymphoblastic leukemia and diffuse large B-cell lymphoma. The tisagenlecleucel manufacturing process was initially developed in an academic setting and subsequently transferred to industry for qualification, validation and scaling up for global clinical trials and commercial distribution. Use of fresh leukapheresis material was recognized early on in the transfer process as a challenge with regard to establishing a global supply chain. To maximize manufacturing success rates and to overcome logistical challenges, cryopreservation was adapted into the Novartis manufacturing process from the beginning of clinical trials. Tisagenlecleucel manufactured in centralized facilities with cryopreserved leukapheresis material has been used successfully in global clinical trials at more than 50 clinical centers in 12 countries. Cryopreservation provides flexibility in scheduling leukapheresis when the patient's health is optimal to provide $\mathrm{T}$ cells; it also provides protection from external factors, such as shipping delays, and removes manufacturing time constraints. Several studies were performed to establish comparability of fresh versus cryopreserved leukapheresis material, to evaluate and optimize the cryopreservation process, to determine the optimal temperature and maximum hold time prior to cryopreservation and to determine the optimal temperature range for shipment and storage. Using the current validated industry manufacturing process, high success rates were achieved with regard to manufacturing tisagenlecleucel batches that met specifications and were released to patients. Consistent product quality and positive clinical outcomes support the use of cryopreserved non-mobilized peripheral mononuclear blood cells collected using leukapheresis for CAR-T cell manufacturing.
\end{abstract}

Key Words: cryopreservation, CTL019 chimeric antigen receptor, immunotherapy, leukapheresis

\section{Introduction}

Chimeric antigen receptor (CAR) - T cell therapy, a cancer immunotherapy in which patients' own immune systems are harnessed to attack tumor cells, has progressed rapidly in the last decade and now represents an important clinical option for certain hematologic malignancies with high unmet need [1-3]. In general, CAR-T manufacturing begins with patients' own $\mathrm{T}$ cells that are genetically modified ex vivo, using lentiviral, retroviral or other gene transfer systems to introduce a CAR transgene [4]. Following infusion into patients, CARs on engineered $\mathrm{T}$ cells bind to antigen-expressing target cells resulting in signal transduction, $\mathrm{T}$-cell activation, in vivo expansion of CAR-T cells and target cell destruction [5]. In clinical trials, CAR-T cell therapies targeting CD19 were effective for treatment of certain B-cell malignancies including B-cell precursor acute lymphoblastic leukemia (B-ALL), chronic lymphocytic leukemia and diffuse large B-cell lymphoma (DLBCL) [6-10]. Tisagenlecleucel (Kymriah) was the first CD19-specific autologous CAR-T cell product approved by the US Food and Drug Administration for treatment of patients aged $\leq 25$ years with relapsed/refractory B-ALL, and later for treatment of adult patients with relapsed/refractory DLBCL after $\geq 2$ lines of systemic therapy [11]. More recently, tisagenlecleucel was approved for

Correspondence: Erik Rutjens, PhD, Novartis Pharma AG, Novartis Technical Operations, NTO Cell and Gene, WST-222, Schaffhauserstrasse 101, 4332 Stein, Switzerland. E-mail: erik.rutjens@novartis.com 
both indications in the European Union [12], Canada (for patients aged 3-25 years) [13], Switzerland [14] and Australia [15].

The tisagenlecleucel CAR is composed of a murine single-chain antibody fragment specific for CD19, a CD8- $\alpha$ hinge and transmembrane region fused to the intracellular $\mathrm{CD} 3-\zeta$ signaling domain, and a 4-1BB co-stimulatory domain (supplementary Figure 1) [5]. CAR-T cells expressing the 4-1BB (CD137) co-stimulatory domain exhibited enhanced CAR-T cell persistence and preferential expansion of memory $\mathrm{T}$ cells when compared with those containing the CD28 co-stimulatory domain or no co-stimulatory domains $[16,17]$.

CAR-T cell manufacturing technology using autologous $\mathrm{T}$ cells for individualized cancer therapy is fundamentally different from previous biological therapies and presents unique challenges. The tisagenlecleucel manufacturing process was initially developed at the University of Pennsylvania (UPenn) in a single-center, single-site trial. This process was then transferred to Novartis, qualified, validated and scaled up for global clinical trials, as well as for commercial distribution [3]. Early in the transfer process, it was recognized that manufacturing processes would need to accommodate routine manufacturing demands and also overcome challenges in establishing a global supply chain of autologous cell and gene therapy products. Using fresh leukapheresis as starting material was identified as a primary challenge. In general, fresh leukapheresis severely limits patient access to a specific geographical region (ruling out a global trial), restricts the time available to commence manufacturing and does not allow for flexibility in scheduling for shipping or patient health status (which could lead to difficulties in apheresing the patient at the required time to match clinical manufacturing slot availability). In singlecenter, single-trial settings, such uncertainty is probably manageable, but can quickly become unmanageable upon moving to multicenter and global trials.

To overcome these limitations, the process was modified to use cryopreserved leukapheresis as starting material. Efforts were made to establish comparability between fresh and cryopreserved leukapheresis from initial transfer studies. Tisagenlecleucel was successfully manufactured in centralized facilities using cryopreserved leukapheresis and was used in two global clinical trials with more than 50 centers in 12 countries for treatment of relapsed/refractory BALL in children and young adults (ELIANA; NCT02435849) [8] and DLBCL in adults (JULIET; NCT02445248) [9]. In this report, we describe the manufacturing process for tisagenlecleucel using cryopreserved leukapheresis as starting material, along with the rationale, supporting studies and benefits of cryopreservation.

\section{Leukapheresis Starting Material: Fresh Versus Cryopreserved}

Leukapheresis is the first step of tisagenlecleucel manufacturing and is arguably very critical because collecting an adequate number of CD3-positive $\mathrm{T}$ lymphocytes is essential to ensure manufacturing success $[18,19]$. Established acceptance criteria specified in the tisagenlecleucel leukapheresis manual (minimum number of CD3-positive lymphocytes and total nucleated cells, and percentage of CD3-positive $T$ cells) must be met for patient leukapheresis material to be accepted for manufacturing. Achieving minimum cell counts can be challenging in patients with leukemia who often have low white blood cell counts [18]. Prior treatment history with cytotoxic therapies can further reduce T-cell numbers. As a result, patients' absolute lymphocyte count and peripheral CD3-positive lymphocyte counts must be determined prior to initiation of leukapheresis. Additional factors, such as correlation between patient weight and volume of blood needed for processing, also play important roles in evaluating patients' ability to undergo leukapheresis [4]. Furthermore, patients' general health at time of leukapheresis is also an important factor contributing to manufacturing success. Therefore, having flexibility to schedule leukapheresis is actually quite beneficial for the overall program, both from a patient perspective and from a manufacturing organization perspective.

When using fresh leukapheresis, there is usually a limited window of time $(24-48 \mathrm{~h})$ within which manufacturing must commence because cell viability decreases over time. Any unforeseen delays in transit of leukapheresis material can reduce the manufacturing success rate. Inability to manufacture leukapheresis material in time will require the patient to be re-leukapheresed, which again becomes highly dependent on patient health status. As can be seen, such events can lead to scheduling complexity.

With fresh leukapheresis, patients must have manufacturing slots reserved for them, or wait for slots to open, before undergoing leukapheresis. This is a challenge because patients could develop an illness before their manufacturing slots, making it difficult or impossible to leukapherese, hence leading to loss of the manufacturing slot altogether. A missed manufacturing slot will likely lead to an inability to use the slot for any other patient because another leukapheresis may not have been scheduled to be received/manufactured at that time. Attempting to reserve another manufacturing slot in the future could create backlogs at manufacturing sites, which may be surmountable, but could result in extremely stressful situations from a patient perspective because time is of the essence in these diseases. 
Cryopreservation provides scheduling flexibility for patients, enabling leukapheresis to occur when the patient's health is optimal for providing healthier $\mathrm{T}$ cells, thus allowing the timing of the procedure to meet the patient's needs rather than those of the manufacturing site. Other advantages of cryopreserved leukapheresis are the ability to accommodate shipping delays and the removal of time constraints prior to manufacturing. To make these scheduling/ logistical challenges seamless for patients and maximize manufacturing success rates, Novartis moved to using cryopreserved patient leukapheresis from the very beginning of clinical trials.

To ensure proper collection, Novartis requires that leukapheresis sites be licensed/accredited with country-specific health authority or accreditation organizations. Sites are trained in leukapheresis and cryopreservation techniques associated with the tisagenlecleucel manufacturing process and are subject to periodic quality control assessments. These procedures are necessary to ensure that patient cells come from qualified sites and meet established acceptance criteria, thus ensuring higher probability of attaining manufacturing success.

\section{Tisagenlecleucel Manufacturing Using Cryopreserved Leukapheresis Material}

Tisagenlecleucel manufacturing (supplementary Figure 2) [4,5] starts with collection of peripheral blood mononuclear cells from patients by leukapheresis $[18,19]$. Leukapheresis material is cryopreserved within $24 \mathrm{~h}$ of collection and shipped to the manufacturing facility to begin the core manufacturing process. At the manufacturing facility, leukapheresis material is thawed using the Barkey Plasmatherm (Barkey, Leopoldshöhe, Germany), an automated dry-thawing system that ensures standardized, temperature-controlled, aseptic thawing of cryopreserved bags. Thawing is followed by cell wash to remove the cryomedium, after which the cells are suspended in a solution containing dextrose and saline and finally in modified expansion media. $\mathrm{T}$ cells are then enriched, selected and activated using anti-CD3/CD28 antibody-coated magnetic beads. The activated $\mathrm{T}$ cells are then transduced using a self-inactivating, minimal lentiviral vector containing the anti-CD19 CAR transgene, and cultured until ready for harvest. Cells are then separated from the magnetic beads, washed, formulated in infusible media and cryopreserved. Once final product release testing is completed, the cryopreserved tisagenlecleucel cells are shipped to infusion sites, where they are thawed, and immediately infused into patients.

\section{Cryopreserved Leukapheresis Material for Tisagenlecleucel Manufacturing: Supporting Studies}

Successful outcomes from comparability studies between UPenn and Novartis, which included fresh and cryopreserved leukapheresis in design and execution, enabled use of cryopreserved leukapheresis in all clinical trials. Additional studies were performed to evaluate and optimize the cryopreservation process, including optimizing temperatures and maximum hold times prior to cryopreservation (time between collection and processing), temperature ranges for shipment and storage and finally the freezing algorithm itself, as described below. All studies described here were sponsored and designed by Novartis Pharmaceuticals, were conducted in accordance with Good Clinical Practice guidelines and the Declaration of Helsinki, and were approved by institutional review boards as appropriate. Healthy donors and patients or their guardians provided written informed consent or assent.

\section{Comparability}

Comparability between fresh and cryopreserved leukapheresis was initially established in site-to-site transfer studies from UPenn to Novartis. Results indicated that products from both fresh and cryopreserved leukapheresis met acceptance criteria and release specifications, and had similar results in characterization assays [20].

A separate comparability study was conducted with healthy donors ( $n=3$ each) to evaluate growth kinetics using fresh and cryopreserved leukapheresis. Comparable cell growth kinetics were observed using both processes (Figure 1). Quality assessments also showed comparable product characteristics (cellular composition, proportion of transduced cells, CAR expression levels and functional response [interferon- $\gamma$ production in response to CD19-expressing target cells]) using both processes (data not shown). These studies confirmed the equivalence of cryopreserved to fresh leukapheresis and laid the foundation for using cryopreserved leukapheresis for all studies.

\section{Hold time and temperature}

Studies were performed to determine the maximum hold time and optimum storage temperature for fresh leukapheresis material prior to cryopreservation in which cell viability was measured over a period of $72 \mathrm{~h}$ for cells stored either at $2-8^{\circ} \mathrm{C}$ or $10-25^{\circ} \mathrm{C}$. Cells stored at $10-25^{\circ} \mathrm{C}$ showed reduced viability as early as $24 \mathrm{~h}$ and deteriorated progressively during the 72 - $\mathrm{h}$ assessment period, whereas cells stored at $2-8^{\circ} \mathrm{C}$ remained viable throughout the 72-h assessment (Figure 2A). Leukapheresis material held at $10-25^{\circ} \mathrm{C}$ for $24-72 \mathrm{~h}$ 


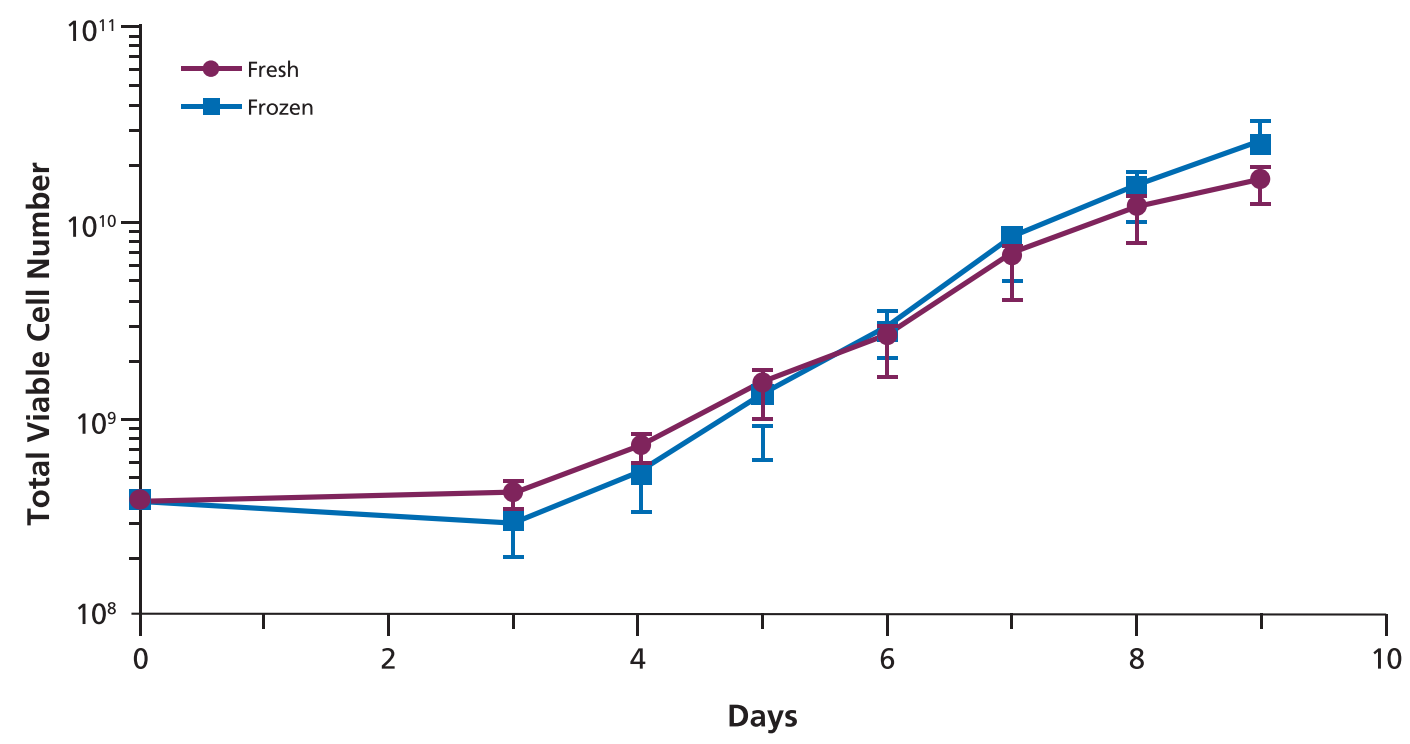

Figure 1. Growth kinetics of fresh versus cryopreserved leukapheresis material from healthy donors $(\mathrm{n}=3$ each). Cell counts were performed on the Multisizer II (Beckman Coulter, Indianapolis, IN, USA) and viability was assessed by microscopy using Trypan Blue exclusion. Total leukocyte counts are reported.

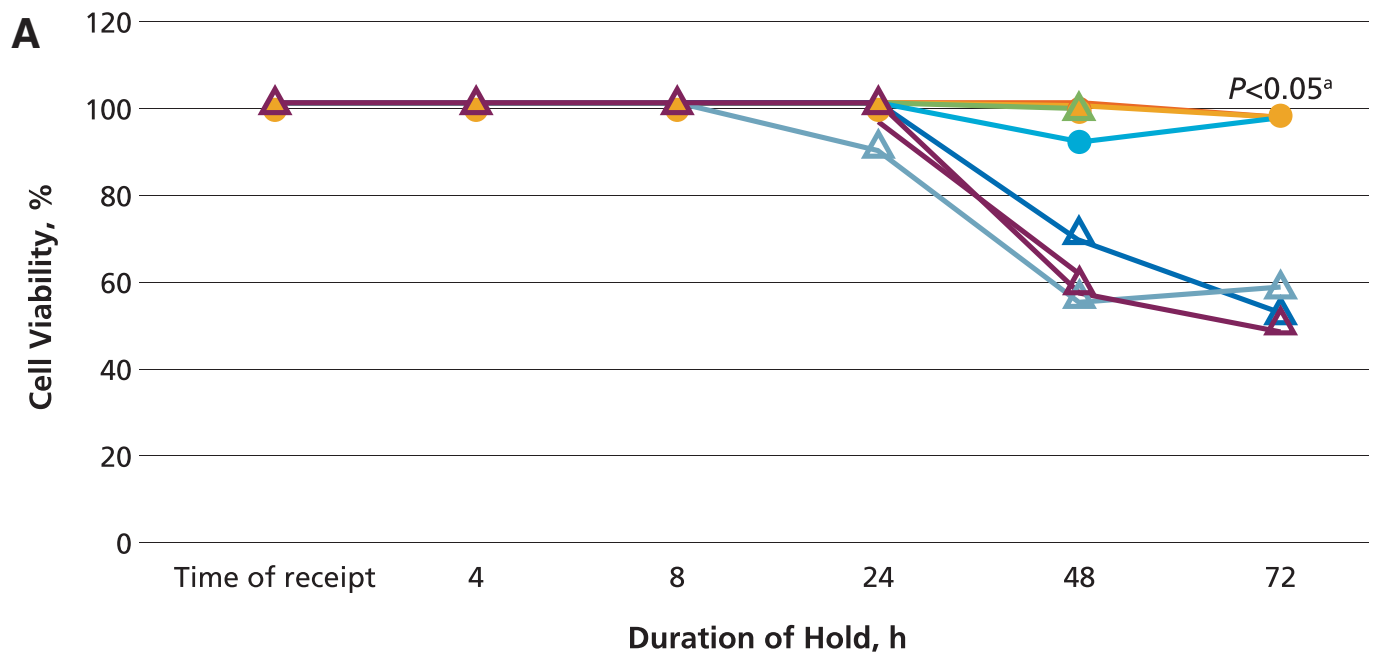

All filled circles represent individual samples held at $2^{\circ} \mathrm{C}-8^{\circ} \mathrm{C}-\Delta-$ All open triangles represent individual samples held at $10^{\circ} \mathrm{C}-25^{\circ} \mathrm{C}$
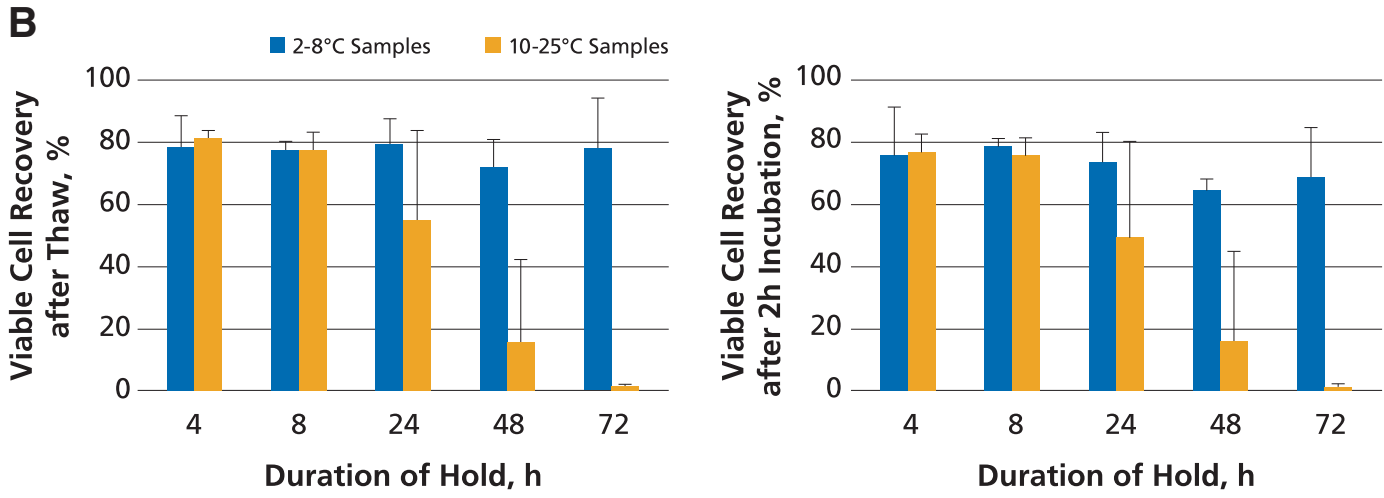

Figure 2. Effect of hold time and temperature $\left(2-8^{\circ} \mathrm{C}\right.$ vs $\left.10-25^{\circ} \mathrm{C}\right)$ on cell viability for $(A)$ fresh leukapheresis material and (B) cryopreserved leukapheresis material. Cell viability was assessed by microscopy using trypan blue exclusion (hemocytometer counts). Viable cell recovery was calculated based on hemocytometer counts using the Multisizer II. ${ }^{a} P<0.05$ between average of samples held at $2-8^{\circ} \mathrm{C}$ vs $10-25^{\circ} \mathrm{C}$ at $72 \mathrm{~h}$. 
Table 1. Summary of internal studies to optimize the cryopreservation process and materials for leukapheresis starting material.

\begin{tabular}{lc}
\hline Study objective & Summary \\
\hline Evaluation of cryobags and cryoformulation & $\begin{array}{c}\text { Leukapheresis material from } 3 \text { individual donors was evaluated using } 10 \text { cryoformulations } \\
\text { and } 4 \text { different cryobags for optimization of the cryopreservation process }\end{array}$ \\
$\begin{array}{l}\text { Evaluation of liquid nitrogen dry vapor cryo- } \\
\text { shippers/shipping conditions for leukaphe- } \\
\text { resis material }\end{array}$ & $\begin{array}{c}\text { demperatures below }-150^{\circ} \mathrm{C} \text { for } \leq 10 \text { days and their impact on leukapheresis material } \\
\text { inside the cryoshippers during real-time shipments using designated courier services }\end{array}$ \\
$\begin{array}{l}\text { Various packaging/shipping conditions were evaluated } \\
\text { primization of controlled-rate freezer }\end{array}$ & $\begin{array}{l}\text { A total of } 40 \text { cryocycles were tested with } 11 \text { normal healthy donor samples } \\
\text { program }\end{array}$ \\
$\begin{array}{c}\text { The program that demonstrated control of the freezing process and good reproducibility } \\
\text { was recommended for cryopreserving leukapheresis material }\end{array}$ \\
\hline
\end{tabular}

before undergoing a freeze-thaw cycle and 2-h culture showed progressively reduced viable cell recovery; whereas material held at $2-8^{\circ} \mathrm{C}$ maintained viable cell recovery post-thaw and post-culture (Figure $2 \mathrm{~B}$ ). Based on these results, cells should be stored at $2-8^{\circ} \mathrm{C}$ prior to cryopreservation to maintain cell health and viability and ensure higher probability of manufacturing success.

\section{Storage time}

Initially, recommended storage time of cryopreserved leukapheresis was determined to be 9 months based upon studies conducted before clinical trial initiation (data not shown). However, additional stability studies conducted to extend storage time indicated that post-thaw stability of leukapheresis stored for 30 months is comparable with that of material stored for 6 weeks, as evidenced by comparable results obtained in cell viability, recovery and phenotype composition (supplementary Figure 3).

\section{Temperature excursions}

Temperature excursions above $-40^{\circ} \mathrm{C}$ reduced viability post-thaw (data not shown), indicating temperature should be maintained in the vapor phase of liquid nitrogen during shipment and storage.

The studies described above further indicated that leukapheresed cells stored for 30 months and protected from temperature excursions can be used successfully in the CAR-T manufacturing process and exhibit efficient $\mathrm{T}$-cell transduction and expansion. Storage time extension from 9 to 30 months has a significant impact on flexibility, both from a manufacturing perspective, but more importantly from a patient and physician perspective as explained earlier.

\section{Additional supporting studies}

The cryopreservation process and materials used for tisagenlecleucel manufacturing (cryobags, cryopreservation medium and controlled-rate freezer program) were optimized and validated in multiple internal studies (Table 1). The current cryoformulation contains $10 \%$ dimethyl sulfoxide and a standard albumin concentration in a physiological electrolyte solution. Shipping logistics and packaging/shipping conditions were also optimized to avoid damage to cryobags and to minimize temperature excursions. Studies included evaluation of specific packaging configurations in cryoshipper containers for the ability to protect cryobags, couriers for their ability to maintain internal temperatures below $-150^{\circ} \mathrm{C}$ for $\leq 10$ days during real-time shipments, design of enclosed cryogenic containers charged with liquid nitrogen and enhanced temperature control mechanisms (Table 1; data not shown).

\section{Clinical outcomes}

Novartis clinical trials were conducted using cryopreserved leukapheresis and the current tisagenlecleucel manufacturing process. Cryopreservation was done using the recommended cocktail for leukapheresis material and the controlled-rate freezer program (although different sites may have used different methods for freezing leukapheresis material), and high success rates were achieved with regard to manufacturing batches that met specifications and were released to patients (supplementary Table 1). Clinical outcomes observed in recent clinical studies (ELIANA and JULIET) [8,9] with tisagenlecleucel manufactured under the current process were generally comparable with early studies that used academic manufacturing processes $[21,22]$.

Based on the currently established manufacturing process, a key advantage of cryopreserved starting materials is the flexibility provided to patients to undergo leukapheresis when timing is most optimal. Collection can be scheduled at patient identification, without waiting for a manufacturing slot. At the physician's discretion, patient cells can be proactively collected before lymphotoxic chemotherapy and can be stored for up to 30 months in advance of tisagenlecleucel manufacturing. The advantage of this situation is that the cells are healthier, less exhausted and probably less senescent and, therefore, will have a greater 
chance of manufacturing success and positive clinical outcome. Optimized shipping containers and shipment methods have been validated to keep cryopreserved leukapheresis material frozen up to 10 days during shipment to the manufacturing facility. All these criteria have enabled and facilitated global shipment and receipt of material, enabling patient access worldwide using centralized manufacturing facilities.

\section{Challenges With Cryopreserved Leukapheresis}

Cryopreserved leukapheresis has several advantages over fresh leukapheresis, and is a "must-have" for globalization of cellular therapy trials. However, with advantages come some challenges, the first of which is storage of cryopreserved material, especially well beyond the traditional shelf-life for leukapheresis material. As the number of clinical trials continues to increase and as more trials reach commercialization, storage space needed for cryopreserved leukapheresis material will continue to increase rapidly, requiring special arrangements for management. The impact will be deeply felt when shelf-life of cryopreserved materials will be measured in years rather than months and when the industry will move toward leukapheresing before administering any chemotherapy, without knowing whether such material will be needed for treatment or not.

Other challenges include the need for standardization of leukapheresis equipment, materials and technology, and the need for harmonized global regulations. Currently, sites follow their respective regional requirements, resulting in site-to-site variability in packaging material and shipping procedures. Leukapheresis material is stored in cryobags of different types and sizes, preventing standardization of shipping conditions. Harmonization of cryobags has its own hurdles. In moving from clinical to commercial stages, this becomes a greater challenge, possibly requiring additional shipping validation studies and/or the need for further standardization of cryobags, both of which may take years to finalize. These challenges should be addressed during the clinical trial stage, ideally through providing "apheresis kits" to sites, rather than during commercialization and launch. Although sites may use different materials and cryopreservation processes, the accreditation and quality control processes described above ensure that these differences will not impact the final product.

Cryopreservation of leukapheresis material is a cellular process inherently subject to freeze-thaw losses in viability and recovery; however, the Novartis apheresis requirements and manufacturing process are robust and were designed with this limitation in mind, and with the knowledge that cryopreservation is necessary to achieve global distribution of a centrally manufactured CAR-T cell therapy product. Indeed, the comparability data discussed in this article demonstrate that the current tisagenlecleucel manufacturing process is able to achieve global distribution. Novartis strives to continue to optimize cryopreservation and processing of leukapheresis material to further minimize the losses associated with this step.

Finally, a certain degree of clumping has been observed with leukapheresis material. If the severity of clumping was determined to have an impact on the manufacturing process, blood filters were used to successfully remove the clumps without further issues, as demonstrated by the viable cell recovery observed following cryopreservation and thaw of leukapheresis material.

\section{Literature Review: Support for Cryopreservation}

In addition to Novartis studies supporting cryopreservation of starting material for CAR-T cell manufacture, other published studies have shown this is a feasible approach. A study evaluating effects of cryopreservation on mononuclear cells used in immunologic assays found no impact of extended storage in liquid nitrogen for $\leq 15$ months on $T$-cell viability or recovery, or on results of lymphocyte proliferation and flow cytometric assays [23]. Other studies compared the impact of cryopreservation on unstimulated cells versus those stimulated with anti-CD3/ anti-CD28 and found that unstimulated cells exhibited decreased post-thaw viability after cryopreservation; however, in stimulated cryopreserved cells, there was no was no impact on viability [24].

Similarly, in subsequent studies by various groups using CAR-T cell products, cryopreservation is being recognized as a logistic necessity as products move from smaller clinical studies into large-scale manufacturing [25]. Pre-clinical studies with fresh versus cryopreserved CD20-specific CAR-T cells showed comparability in phenotype (cell types and activation, differentiation and exhaustion markers) and function (interferon- $\gamma$, interleukin-2 and tumor necrosis factor $-\alpha$ secretion) [26]. In an analysis of multiple CAR- $T$ cell products manufactured in different clinical trials (160 CAR-T cell cultures/products initiated for patient infusion across six clinical trials), products manufactured after initial cryopreservation met clinical dose requirements, and there was no impact of cryopreservation on fold expansion, transduction efficiency, percentage of CD3-positive cells or CD4/CD8 ratios in manufactured CAR-T cells [25]. For anti-CD19 CAR-T cell product axicabtagene ciloleucel, fresh product was used in initial clinical trials [27]; however, in a subsequent phase 1 
study, ZUMA-1, cryopreserved product was used and the study reported comparable expansion and clinical responses as seen with fresh product $[28,29]$.

As is evident from the above discussion, from logistical necessity and need for flexibility, to minimal impact on post-thaw viability or performance, cryopreserved leukapheresis is gaining importance and is moving toward a "must have" for global supply of clinical and commercial cell and gene therapy products. In addition, technologies and systems used for shipping materials in liquid nitrogen containers have developed greatly over the last several years, facilitating transportation and logistics.

Further, as it is still early in the cell and gene therapy expansion era, manufacturing facilities are currently limited by geographical regions and manufacturing capacity. Therefore, it is imperative that the industry itself move to cryopreserved leukapheresis, to match manufacturing capacity with global demand. This was also the experience in the ZUMA trials with axicabtagene ciloleucel; clinical studies were initially performed with fresh leukapheresis, but subsequent trials have moved to introducing cryopreservation steps in the manufacturing process.

We have presented both the difficulties and advantages of cryopreserving patient cells. Since Novartis has used cryopreserved leukapheresis from the very beginning, all clinical data have been derived from cryopreserved leukapheresis. As discussed above, clinical outcomes using the current manufacturing process $[8,9]$ are comparable with those in UPenn trials [21,22], which used a mixture of fresh and frozen apheresis.

\section{Conclusion}

The rationale for using cryopreserved leukapheresis material in the tisagenlecleucel manufacturing process stems from identification of logistical challenges associated with using fresh leukapheresis material during scale up from single-center to global trials. Cryopreservation addresses these challenges by providing multiple benefits, the most important being improved flexibility in terms of scheduling leukapheresis when timing is optimal for patients. Comparability between fresh and cryopreserved leukapheresis was established in early studies during transfer from academia to industry, and several additional studies were performed to evaluate and optimize the cryopreservation process for leukapheresis starting material. The resulting robust, validated industry manufacturing process for tisagenlecleucel produces consistent product quality and positive clinical outcomes that support the use of cryopreserved leukapheresed cells as the starting material for CAR-T cell manufacturing.

\section{Declaration of Competing Interest}

The studies and writing assistance were funded by Novartis Pharmaceuticals Corporation, East Hanover, NJ, USA. S.T., C.A. and E.R. are employees of Novartis Pharmaceuticals Corporation, East Hanover, NJ, USA. D.S. is an employee of Novartis Pharma AG, Basel, Switzerland. The sponsor was involved in the collection, analysis and interpretation of data, in the writing of the report and in the decision to submit the article for publication.

\section{Author Contributions}

All authors have contributed to the analysis and interpretation of data and drafting or revising the manuscript, and have approved the final article.

\section{Acknowledgments}

The authors acknowledge the contributions of Dr. Elizabeth Pratico for discussion of data and critical review of the manuscript. Medical writing assistance was provided by Beena John, PhD, of C4 MedSolutions, LLC (Yardley, PA, USA), a CHC Group company.

\section{References}

[1] Maus MV, Levine BL. Chimeric antigen receptor T-cell therapy for the community oncologist. Oncologist 2016;21:608-17.

[2] Wang X, Riviere I. Clinical manufacturing of CAR T cells: foundation of a promising therapy. Mol Ther Oncolytics 2016;3:16015.

[3] Levine BL, Miskin J, Wonnacott K, Keir C. Global manufacturing of CAR T cell therapy. Mol Ther Methods Clin Dev 2017;4:92-101.

[4] McGuirk J, Waller EK, Qayed M, Abhyankar S, Ericson S, Holman $\mathrm{P}$, et al. Building blocks for institutional preparation of CTL019 delivery. Cytotherapy 2017;19(9):1015-24.

[5] Buechner J, Kersten MJ, Fuchs M, Salmon F, Jäger U. Chimeric antigen receptor- $\mathrm{T}$ cell therapy: practical considerations for implementation in Europe. HemaSphere 2018;2(1):e18.

[6] Kalos M, Levine BL, Porter DL, Katz S, Grupp SA, Bagg A, et al. $T$ cells with chimeric antigen receptors have potent antitumor effects and can establish memory in patients with advanced leukemia. Sci Transl Med 2011;3(95). $95 \mathrm{ra} 73$.

[7] Porter DL, Hwang WT, Frey NV, Lacey SF, Shaw PA, Loren AW, et al. Chimeric antigen receptor $\mathrm{T}$ cells persist and induce sustained remissions in relapsed refractory chronic lymphocytic leukemia. Sci Transl Med 2015;7(303). 303ra139.

[8] Maude SL, Laetsch TW, Buechner J, Rives S, Boyer M, Bittencourt $\mathrm{H}$, et al. Tisagenlecleucel in children and young adults with B-cell lymphoblastic leukemia. N Engl J Med 2018;378(5):439-48.

[9] Schuster SJ, Bishop MR, Tam CS, Waller EK, Borchmann P, McGuirk JP, et al. Tisagenlecleucel in Adult Relapsed or Refractory Diffuse Large B-Cell Lymphoma. N Engl J Med 2019;380(1):45-56.

[10] Neelapu SS, Locke FL, Bartlett NL, Lekakis LJ, Miklos DB, Jacobson CA, et al. Axicabtagene ciloleucel CAR T-cell therapy in refractory large B-cell lymphoma. N Engl J Med 2017;377(26):2531-44. 
[11] KYMRIAH (tisagenlecleucel). Full Prescribing Information. East Hanover, NJ: Novartis Pharmaceuticals Corporation; 2018.

[12] Kymriah (tisagenlecleucel). Summary of Product Characteristics. Dublin, Ireland: Novartis Europharm Limited; 2018.

[13] Kymriah (tisagenlecleucel). Health Canada Product Monograph. Dorval, Quebec, Canada: Novartis Pharmaceuticals Canada Inc.; 2018.

[14] KymriahTM, Zellsuspension zur Infusion (Tisagenlecleucelum). https://www.swissmedic.ch/swissmedic/de/home/humanarzneimittel/authorisations/new-medicines/kymriahtm_zellsuspensionzurinfusiontisagenlecleucelum.html. [accessed February 20, 2019].

[15] Kymriah (tisagenlecleucel). Australian Prescribing Information. Macquarie Park, NSW, Australia: Novartis Pharmaceuticals Australia Pty Ltd; 2018.

[16] Milone MC, Fish JD, Carpenito C, Carroll RG, Binder GK, Teachey D, et al. Chimeric receptors containing CD137 signal transduction domains mediate enhanced survival of $\mathrm{T}$ cells and increased antileukemic efficacy in vivo. Mol Ther 2009;17(8):1453-64.

[17] Zhang H, Snyder KM, Suhoski MM, Maus MV, Kapoor V, June $\mathrm{CH}$, et al. $4-1 \mathrm{BB}$ is superior to $\mathrm{CD} 28$ costimulation for generating CD8+ cytotoxic lymphocytes for adoptive immunotherapy. J Immunol 2007;179(7):4910-8.

[18] Allen ES, Stroncek DF, Ren J, Eder AF, West KA, Fry TJ, et al. Autologous lymphapheresis for the production of chimeric antigen receptor T cells. Transfusion 2017;57(5):1133-41.

[19] Fesnak A, Lin C, Siegel DL, Maus MV. CAR-T cell therapies from the transfusion medicine perspective. Transfus Med Rev 2016;30(3):139-45.

[20] Boyd JA, Levine BL, Jinivizian K, Jeschke MA, Suhoski Davis MM, Zheng Z, et al. Successful translation of chimeric antigen receptor (CAR) targeting CD19 (CTL019) cell processing technology from academia to industry. Blood 2015;126(23):3100.

[21] Maude SL, Frey N, Shaw PA, Aplenc R, Barrett DM, Bunin NJ, et al. Chimeric antigen receptor $\mathrm{T}$ cells for sustained remissions in leukemia. N Engl J Med 2014;371 (16):1507-17.

[22] Schuster SJ, Svoboda J, Chong EA, Nasta SD, Mato AR, Anak $\mathrm{O}$, et al. Chimeric antigen receptor $\mathrm{T}$ cells in refractory B-cell lymphomas. N Engl J Med 2017;377(26):2545-54.
[23] Weinberg A, Song LY, Wilkening C, Sevin A, Blais B, Louzao R, et al. Optimization and limitations of use of cryopreserved peripheral blood mononuclear cells for functional and phenotypic T-cell characterization. Clin Vaccine Immunol 2009;16(8):1176-86.

[24] Jeurink PV, Vissers YM, Rappard B, Savelkoul HF. T cell responses in fresh and cryopreserved peripheral blood mononuclear cells: kinetics of cell viability, cellular subsets, proliferation, and cytokine production. Cryobiology 2008;57(2):91103.

[25] Elavia N, McManus A, Highfill SL, Ren J, Shah NN, Fry TJ, et al. The post-thaw recovery of cryopreserved chimeric antigen receptor (CAR) T-cells during manufacture is better than that of cryopreserved peripheral blood CD3+ cells. Blood 2017;130(Suppl 1):4475.

[26] Lee SY, Olsen P, Lee DH, Kenoyer AL, Budde LE, O'Steen $\mathrm{S}$, et al. Preclinical optimization of a CD20-specific chimeric antigen receptor vector and culture conditions. J Immunother 2018;41(1):19-31.

[27] Kochenderfer JN, Dudley ME, Kassim SH, Somerville RP, Carpenter RO, Stetler-Stevenson M, et al. Chemotherapyrefractory diffuse large B-cell lymphoma and indolent B-cell malignancies can be effectively treated with autologous $\mathrm{T}$ cells expressing an anti-CD19 chimeric antigen receptor. J Clin Oncol 2015;33(6):540-9.

[28] Locke FL, Neelapu SS, Bartlett NL, Siddiqi T, Chavez JC, Hosing CM, et al. Phase 1 results of ZUMA-1: a multicenter study of KTE-C19 anti-CD19 CAR T cell therapy in refractory aggressive lymphoma. Mol Ther 2017;25(1):285-95.

[29] Roberts ZJ, Better M, Bot A, Roberts MR, Ribas A. Axicabtagene ciloleucel, a first-in-class CAR $\mathrm{T}$ cell therapy for aggressive NHL. Leuk Lymphoma 2017:1-12.

\section{Supplementary materials}

Supplementary material associated with this article can be found in the online version at doi:10.1016/j. jcyt.2019.10.005. 